ORIGINAL ARTICLE

\title{
Mechanisms of cognitive-behavioral therapy for obsessive-compulsive disorder involve robust and extensive increases in brain network connectivity
}

\author{
TD Moody ${ }^{1}$, F Morfini ${ }^{1}$, G Cheng ${ }^{1}$, C Sheen ${ }^{1}$, R Tadayonnejad ${ }^{1}$, N Reggente ${ }^{2}$, J O’Neill ${ }^{1}$ and JD Feusner ${ }^{1}$
}

Cognitive-behavioral therapy (CBT) is effective for obsessive compulsive disorder (OCD); however, little is understood about its mechanisms related to brain network connectivity. We examined connectivity changes from resting-state functional magnetic resonance imaging data pre-to-post-CBT in 43 OCD participants, randomized to receive either 4 weeks of intensive CBT or 4 weeks waitlist followed by 4 weeks of CBT, and 24 healthy controls before and after 4 weeks of no treatment. Network-based-statistic analysis revealed large-magnitude increases in OCD connectivity in eight networks. Strongest increases involved connectivity between the cerebellum and caudate/putamen, and between the cerebellum and dorsolateral/ventrolateral prefrontal cortices. Connectivity increases were associated with increased resistance to compulsions. Mechanisms of CBT may involve enhanced cross-network integration, both within and outside of classical cortico-striatal-thalamo-cortical regions; those involving cerebellar to striatal and prefrontal regions may reflect acquisition of new non-compulsive goal-directed behaviors and thought patterns. Our findings have implications for identifying targets for enhancing treatment efficacy and monitoring treatment progress.

Translational Psychiatry (2017) 7, e1230; doi:10.1038/tp.2017.192; published online 5 September 2017

\section{INTRODUCTION}

Obsessive-compulsive disorder $(\mathrm{OCD})^{1}$ is a common, distressing, and impairing ${ }^{2}$ condition characterized by recurrent intrusive, disturbing thoughts (obsessions) and/or stereotyped behaviors (compulsions). Cognitive-behavioral therapy (CBT), ${ }^{3-5}$ especially intensive $\mathrm{CBT}^{6,7}$ is a well established, but often incompletely effective treatment for OCD. Elucidation of the brain mechanisms of CBT could enhance existing treatments and open new routes to improved treatment response.

Brain effects of CBT are incompletely explored. Functional magnetic resonance imaging (fMRI) and positron emission tomography (PET) indicate that CBT attenuates pathophysiological hyperactivity in classical cortico-striato-thalamo-cortical (CSTC) OCD regions, such as caudate, putamen, thalamus, anterior cingulate cortex (ACC) and orbitofrontal cortex (OFC) ${ }^{8,9}$ Other studies, however, revealed OCD pathophysiology outside classical regions. ${ }^{10-14}$ It is plausible that extra-CSTC actions of CBT remediate OCD by normalizing CSTC-hyperactivity or via independent compensatory mechanisms. Such effects represent potential targets for improved treatment efficacy.

Advances in neuroimaging of functional brain connectivity offer opportunities to explore CSTC and extra-CSTC effects of CBT. Analyzing resting-state blood-oxygenation-level dependent (BOLD) functional connectivity using graph theory, one study reported that serotonin reuptake inhibitor (SRI) treatment of OCD increased the global brain network metrics small-worldness and clustering coefficient (indices of information-transfer efficiency), and induced frontal/parietal increases and occipital decreases in local connectivity. ${ }^{15}$ Similarly, our earlier study, focused on prediction of OCD relapse after $\mathrm{CBT}^{16}{ }^{16}$ found pre-to-post-CBT increases in small-worldness and clustering coefficient.
The present study aimed to deepen understanding of the mechanisms of CBT in OCD by measuring pre-to-post-CBT changes in functional connectivity. Our focus was on identifying regional, rather than global, pre-to-post network connectivity changes and on determining whether such changes were associated with improved clinical symptoms.

Functional connectivity from resting-state fMRI (rsfMRI) was evaluated before and after 4 weeks of intensive CBT, including comparison to OCD-waitlist and healthy control groups scanned at corresponding time points. For the analysis we used the networkbased statistic (NBS) method. Compared to mass-univariate testing, NBS has greater statistical power in examining local network effects, as it accounts for the extent to which the connections involved in the effect of interest (here, CBT) are interconnected. ${ }^{17}$ We employed this data-driven approach because effects of CBT on local functional connectivity in OCD have not previously been tested. Notwithstanding, based on the two previous connectivity studies of OCD treatment ${ }^{15,16}$ and on CBT functional connectivity studies in other disorders, ${ }^{18-23}$ we predicted CBT to result in connectivity increases in the OCD group in networks that cross several classical (caudate and putamen) ${ }^{20}$ and non-classical (inferior frontal gyrus, ${ }^{23}$ middle frontal gyrus, ${ }^{15,18,19,21,22}$ precentral gyrus, ${ }^{15}$ insula, ${ }^{15,21,22}$ posterior cingulate cortex, ${ }^{15}$ occipital cortex, ${ }^{15}$ and cerebellum ${ }^{19,22}$ ) sites. Strengthening connections with frontal cortices is thought to improve cognitive control and top-down modulation of emotions. Modifying connectivity across other regions may relate to improvements in functions relevant to OCD psychopathology, including switching between default mode and executive action (insula ${ }^{13}$ ), assessment of self-relevant stimuli (posterior cingulate ${ }^{11}$ ), visuospatial functioning (occipital cortex), ${ }^{24,25}$ and

\footnotetext{
${ }^{1}$ Department of Psychiatry and Biobehavioral Sciences, David Geffen School of Medicine at UCLA, Los Angeles, CA, USA and ${ }^{2}$ Department of Psychology, University of California Los Angeles, Los Angeles, CA, USA. Correspondence: Dr TD Moody, UCLA Semel Institute, Box 951759, Westwood Boulevard 27-465, Los Angeles, CA 90095-1759, USA. E-mail: tmoody@ucla.edu

Received 17 May 2017; accepted 13 June 2017
} 
compulsion-related motor control and modulation of higher-order cognition and affect (cerebellum). ${ }^{26}$ However, within CSTC circuits we predicted that connectivity would be stronger between the caudate and the OFC on the right and the left in OCD compared with healthy controls, based on previous studies ${ }^{27-29}$ and that this connectivity would decrease with treatment. We also predicted that increases with treatment in connectivity in these aforementioned networks and between caudate and OFC would be associated with improved OCD symptoms.

\section{MATERIALS AND METHODS}

Recruitment/assessment

We recruited participants through UCLA clinics, flyers, and internet advertisements. All provided informed consent with UCLA Institutional Review Board approval. OCD diagnosis was established through interviews by one author (JDF), who has clinical experience with this population. Primary OCD and comorbid diagnoses were determined using the ADIS-IVMini. $^{30}$ OCD participants were eligible if they scored 16 on the Yale-Brown Obsessive Compulsive Scale (YBOCS) ${ }^{31}$ and had OCD age-of-onset before 18 years. Exclusion criteria for OCD included psychotic disorders, bipolar disorder, lifetime substance dependence, or attention-deficit hyperactivity disorder (ADHD). Comorbid anxiety and depressive disorders were allowed if OCD was the primary diagnosis; however, individuals were excluded if the ADIS-IV clinical significance rating for depression was $\geqslant 6$ (severe). To recruit a clinically representative OCD sample, individuals who were either unmedicated or taking SRIs only were included, but no changes in medication within 12 weeks prior to enrollment were allowed. We excluded those with $\geqslant 30$ sessions of prior CBT to minimize the possibility of brain changes induced by previous CBT. Exclusion criteria for healthy controls included psychiatric disorders (including specific phobias) per ADIS-IV-Mini, or psychiatric medications. Exclusion criteria for OCD and controls included IQ $<80$ on the Wechsler Abbreviated Scales of Intelligence (WASI) ${ }^{32}$ and medical conditions affecting cerebral metabolism (for example, thyroid disorders and diabetes).

\section{Randomization and blinding}

For randomization of OCD participants to treatment-first or minimal contact waitlist-first followed by treatment, we used randomized permuted blocking ${ }^{33}$ with block size of 4 and covariate-adaptive randomization for medication status, gender and age. ${ }^{34}$ Independent evaluators not involved in treatment or assessments administered psychometric instruments (Supplementary Protocol 1). Estimated reliability between evaluators was high (Intraclass Correlation Coefficient 0.74).

\section{Psychometric evaluations}

Primary outcome was the YBOCS. ${ }^{31}$ Secondary measures included the Obsessive Compulsive Inventory Revised (OCI-R), ${ }^{35}$ Hamilton Anxiety Scale $(\text { HAMA })^{36}$ and the Montgomery-Åsberg Depression Rating Scale (MADRS). ${ }^{37}$ General functionality and social/occupational performance was rated with the Global Assessment Scale (GAS). ${ }^{38}$

\section{Treatment}

All OCD participants underwent manualized ${ }^{39}$ exposure and response prevention (ERP)-based intensive CBT with individual therapists (90-minute sessions, 5 days per week, 4 weeks). Two licensed therapists with extensive training in CBT for OCD conducted treatment. Both had 6 or more years of specialty training in intensive and outpatient CBT for OCD. Therapy sessions of consenting participants (61\%) were videotaped, and an independent evaluator (also a trained CBT therapist with 8 years experience) rated all sessions for quality assurance, including adherence to the manual and overall quality of the session (Supplementary Protocol 1). Average treatment adherence was $97.7 \%$ and average quality of sessions was 9.96 (0-10 scale).

\section{FMRI acquisition and processing}

MRI data were acquired before and after 4-week intervals at 3T (Siemens Trio, Los Angeles, CA, USA) with a 12-channel head coil. Whole-brain BOLD fMRI was collected using a 7-min echo-planar imaging (EPI) sequence $\left(T R / T E=2000 / 25 \mathrm{~ms}\right.$, flip angle $=78^{\circ}$, voxels $3 \mathrm{~mm}^{3}, 1-\mathrm{mm}$ gap).

\section{Preprocess rsfMRI data}

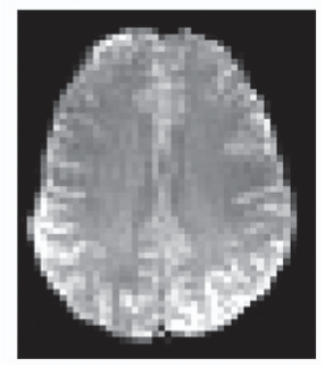

2. Define nodes

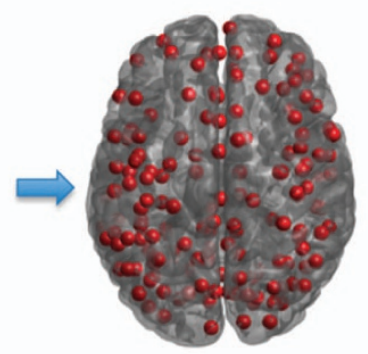

NBS METHODS

3. Extract time series

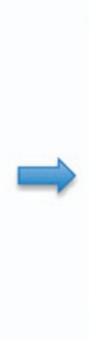

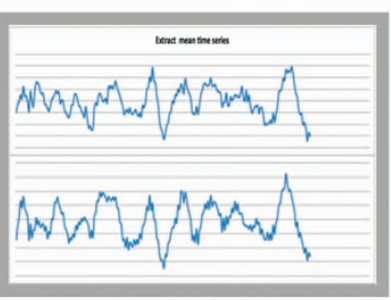

4. Calculate functional connectivity

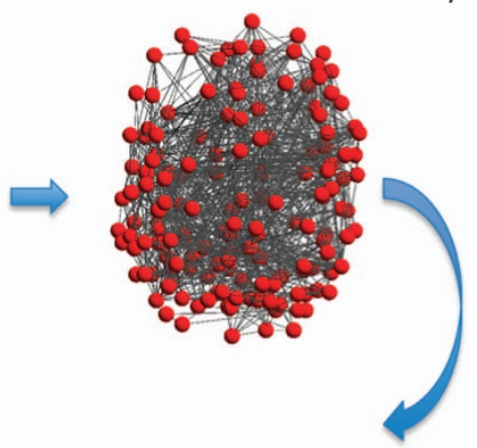

7. Linear regressions

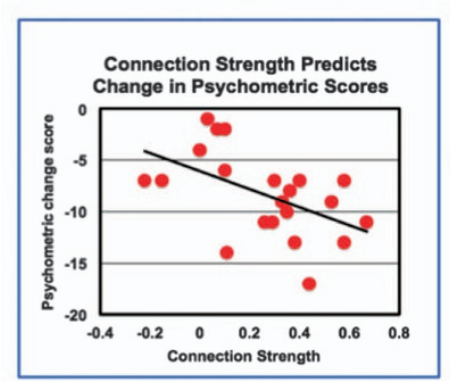

6. FDR: identify connections

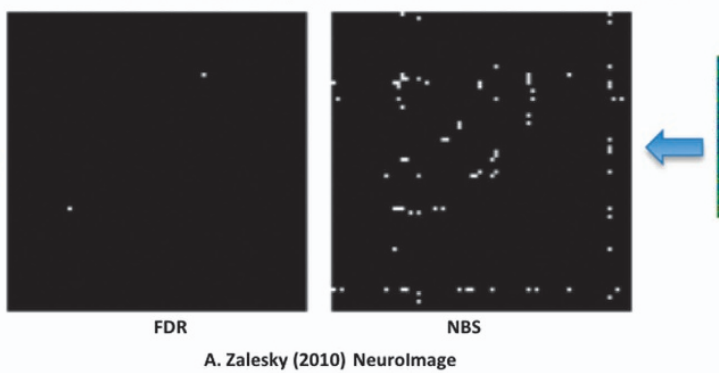

5. Create functional connectivity matrices

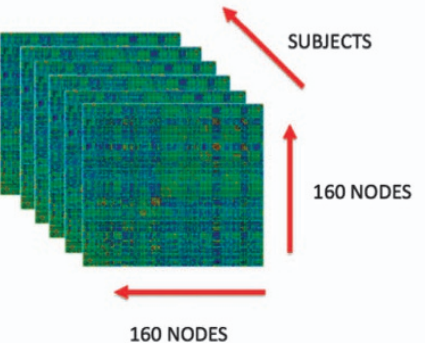

Figure 1. Flowchart for NBS analysis of whole-brain resting-state fMRI. (1) Resting state data were acquired, preprocessed to remove motion artifact, and normalized to the MNI template; (2) On the MNI template, 160 functional nodes were defined; (3) Time series within each node were extracted; (4) Functional connectivity was calculated for each pair of nodes for each participant; (5) $160 \times 160$ connectivity matrices (fullcorrelations) were computed; (6) Network-based statistic (NBS) method was used to identify connectivity differences between groups and before and after CBT; (7) Linear regressions compared connectivity strength and OCD symptoms. rsfMRI, resting state functional MRI. 
Participants were instructed to rest with eyes closed and not to sleep. Whole-brain T1-weighted MRI (MPRAGE, TR/TE $=1900 / 3.26 \mathrm{~ms}$, voxels $1 \mathrm{~mm}^{3}$ ) was acquired for registration.

Functional data were preprocessed using FSL 5.0.4 (http://www.fmrib.ox. ac.uk/fsl). Data were motion-corrected (MCFLIRT) and band-pass filtered $(0.009-0.08 \mathrm{~Hz})$. Seven and twelve degrees-of-freedom transforms were used to register functional images to MPRAGE and to Montreal Neurological Institute (MNI) space, respectively, and data were resampled to 2-mm space. Preprocessing included intensity normalization, but not spatial smoothing, and linear regression of global signal, cerebrospinal fluid, and their first derivatives, as well as six head-motion parameters. Motion was assessed using DVARS (root-mean-squared change in volumeto-volume BOLD signal). ${ }^{40}$ There were no significant differences in DVARS for pre-CBT $(29.2 \pm 4.0)$ vs post-CBT $(28.6 \pm 4.7)$ OCD $(P=0.48$, paired $t$-test $)$ or for Week $1(28.1 \pm 3.2)$ vs Week $4(29.5 \pm 4.0)$ controls $(P=0.10)$, nor were there any between-group differences at either timepoint: OCD vs controls: Week $1(29.2 \pm 4.4)$ vs $(28.1 \pm 3.2),(P=0.29$; independent $t$-test); Week 4 $(28.6 \pm 4.7)$ vs $(29.5 \pm 4.9),(P=0.42)$.

\section{Connectivity analyses}

Network-based statistic analysis. We sampled the entire brain using 160 functionally defined, non-overlapping, $10-\mathrm{mm}$ diameter nodes (Figure 1). ${ }^{41}$ We calculated all pairwise full-correlation coefficients, without thresholding, allowing all positive and negative values, between nodes for each individual's rsfMRI time-series data. NBS is data-driven and allows one to identify functionally correlated networks of brain regions. Specific subsets of nodes are determined to be statistically significant based on mutual connections in topological rather than physical space. We used the NBS Toolbox ${ }^{17}$ to analyze functional matrices with statistical threshold $P=0.01$, 10000 permutations, $t$-threshold $=6$, and exchange blocks for paired comparisons. Given the arbitrary choice of threshold, we retested at lower and higher thresholds (Supplementary Figure 1, Supplementary Table 1 and Supplementary Video 1). For OCD vs control comparisons, medicationstatus and randomization-arm (treatment-first or waitlist-first) regressors were added to the model, with $P=0.05,10000$ permutations, and a lower t-threshold $=4$, given the lower power for $n=24$ controls. For both comparisons, networks were identical using extent or intensity thresholding.

CSTC nodal connectivity analysis. Because the widely used node set identified by Dosenbach ${ }^{41}$ did not include specific CSTC regions of interest to us, we performed an additional nodal functional connectivity analysis implemented with the REST toolbox (http://www.rest.restfmri.net) to investigate CTSC nodes. We limited our investigation to 7 nodes identified from the Neurosynth database (http://www.neurosynth.org, a platform for synthesis of fMRI data from $>11000$ studies with meta-analyses of $>300$ terms and 150000 brain locations) using the search term 'Obsessive Compulsive', and then analyzed differences in functional connectivity between $O C D$ and $H C$ at baseline and for OCD before and after CBT. We used 2-sample t-tests for OCD vs HC comparisons, and paired $t$-tests for pre- vs post CBT connectivity in OCD. A Bonferroni correction for multiple comparisons was employed for testing the hypotheses involving right and left ipsilateral connections between caudate and OFC ( $P$-value $\leqslant 0.025)$, and a false-discovery rate correction (FDR) for the remaining exploratory hypotheses for all other node pairs in the 7-node set. Detailed methods of nodal connectivity analysis can be found in the Supplementary Materials.

Data analysis and associations with clinical variables

We used paired $t$-tests (SPSS V23, IBM, Armonk, NY, USA) to assess treatment response and linear regressions to test relationships between changes in connection strength and changes in psychometric scores. Regressions evaluating changes in OCD symptoms and network connectivity with treatment examined 18 connections at $P=0.05$, Bonferronicorrected to $P=0.05 / 18=0.0028$. We conducted exploratory analyses to examine whether connection strength prior to CBT was associated with changes in OCD symptoms (YBOCS), anxiety (HAMA) and depression (MADRS).

\section{RESULTS}

We assessed 76 participants (Table 1, Supplementary Figure 2). Fifty-one right-handed adults ages 18-60 years diagnosed with
Table 1. Obsessive-compulsive disorder and healthy control samples

\begin{tabular}{|c|c|c|c|}
\hline \multirow[t]{2}{*}{ Characteristics } & $O C D$ & Control & \multirow[t]{2}{*}{ P-value } \\
\hline & $(n=43)$ & $(n=24)$ & \\
\hline Female/male & $21 / 22$ & $10 / 14$ & $0.57^{\mathrm{a}}$ \\
\hline Age (s.d.) & $33(10.7)$ & $31(12.0)$ & $0.49^{\mathrm{b}}$ \\
\hline Education (s.d.), years & $15.6(2.4)$ & $15.4(2.3)$ & $0.74^{b}$ \\
\hline WASI IQ (s.d.) & $108.2(9.1)$ & $109(8.8)$ & $0.73^{\mathrm{b}}$ \\
\hline GAS (s.d.) & $57.6(8.5)$ & $84.8(19.2)$ & $<0.001^{\mathrm{b}}$ \\
\hline Serotonin-reuptake inhibitor & 14 & & \\
\hline \multicolumn{4}{|l|}{ Psychiatric comorbidities } \\
\hline None & 12 & & \\
\hline Panic disorder & 2 & & \\
\hline Generalized anxiety disorder & 9 & & \\
\hline Social anxiety disorder & 17 & & \\
\hline Major depressive disorder & 7 & & \\
\hline Dysthymia & 2 & & \\
\hline Body dysmorphic disorder & 4 & & \\
\hline Post-traumatic stress disorder & 1 & & \\
\hline Specific phobia & 6 & & \\
\hline $\begin{array}{l}\text { Depressive disorder not } \\
\text { otherwise specified }\end{array}$ & 1 & & \\
\hline YBOCS total pre-CBT & $24.5(4.7)$ & & \\
\hline YBOCS total post-CBT & $15.0(5.2)$ & & $<0.001^{c}$ \\
\hline OCI-R pre-CBT & $1.53(1.0)$ & & \\
\hline OCI-R post-CBT & $0.92(0.8)$ & & $<0.001^{c}$ \\
\hline HAMA pre-CBT & $12.4(5.4)$ & & \\
\hline HAMA post-CBT & $8.4(5.1)$ & & $<0.001^{\circ}$ \\
\hline MADRS pre-CBT & $15.3(9.5)$ & & \\
\hline MADRS post-CBT & $10.8(8.9)$ & & $<0.001^{\circ}$ \\
\hline GAS pre-CBT & $57.6(8.5)$ & & \\
\hline GAS post-CBT & $69.6(13.3)$ & & $<0.001^{c}$ \\
\hline
\end{tabular}

Abbreviations: CBT, cognitive-behavioral therapy; GAS, Global Assessment Scale; HAMA, Hamilton Anxiety Scale; MADRS, Montgomery-Åsberg Depression Rating Scale; OCD, obsessive compulsive disorder; OCI-R, Obsessive Compulsive Inventory Revised; WASI, Wechsler Abbreviated Scales of Intelligence; YBOCS, Yale-Brown Obsessive Compulsive Scale. See Supplementary Table 2 for complete YBOCS item score pre- and post-CBT. ${ }^{a}$ Chi-squared test. ${ }^{\mathrm{b}}$ Independent $t$-test. ${ }^{\text {CPaired }} t$-test, comparing preversus post-CBT.

DSM-IV OCD were randomized. Four waitlist-first participants elected to withdraw before finishing waitlist and 1 was withdrawn due to medication protocol violation. The study physician withdrew 2 treatment-first participants, and 1 completed the study but had inadequate data due to head motion. Twenty-five healthy controls ages 19-60 years participated; one had inadequate data due to head motion. Ultimately, 43 OCD and 24 controls were analyzed. Symptom dimensions for OCD participants at baseline are presented in Supplementary Figure 3.

Within the OCD sample, six patients were undergoing concurrent treatment with fluoxetine, one with fluvoxamine, two with escitalopram, three with sertraline and two with paroxetine. The proportion of OCD participants on medication did not differ between the active CBT and waitlist arms $\left(x^{2}=1.43, P=0.23\right)$.

Pre- to post-CBT and waitlist OCD symptom changes YBOCS improved pre-to-post-CBT for all but one patient (pre-CBT mean: $24.5 \pm 4.7$, post-CBT: $15.0 \pm 5.2$; improvement $9.7 \pm 5.8$ (39.6\%): $t=11.0, P<0.001$; Table 1). YBOCS Obsessions subscale improved from $11.9 \pm 2.7$ to $7.9 \pm 3.1(4.0 \pm 3.3$ (33.4\%) improvement: $t=7.94, P<0.001)$. YBOCS Compulsions subscale improved 


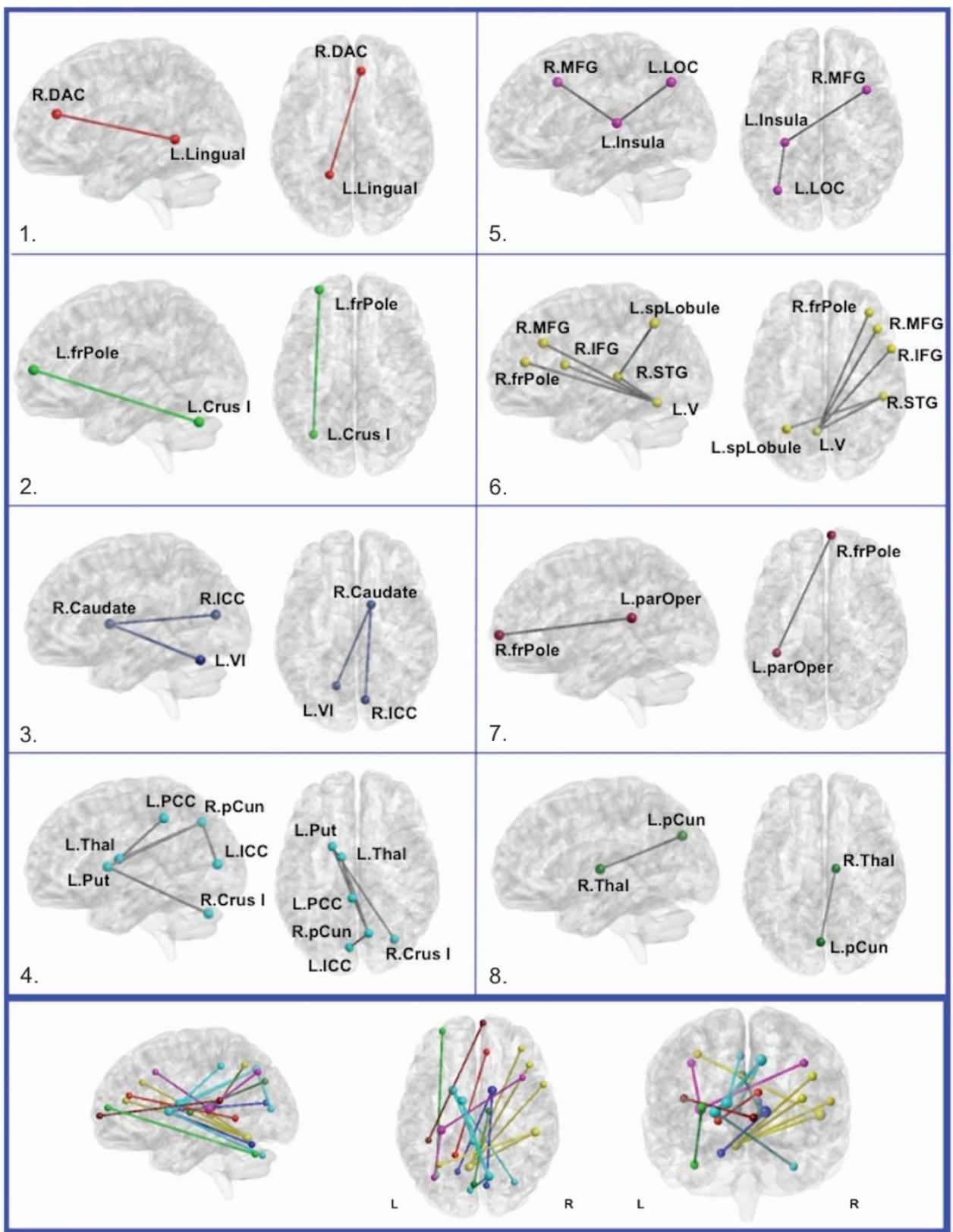

Figure 2. Obsessive compulsive disorder (OCD) networks showing significantly stronger connectivity pre- to post-CBT. L, left; R, right; L.Crus I, L. cerebellum crus I; L.frPole, L. frontal pole; L.ICC, L. intracalcarine cortex; L.Insula, L. posterior insula; L.Lingual, L. lingual gyrus; L.LOC, L. superior lateral occipital; L.parOper, L. parietal operculum; L.PCC, L. posterior cingulate; L.pCun, L. precuneus; L.Put, L. putamen; L.spLobule, L. superior parietal lobule; L.Thal, L. thalamus, caudate; L.V, L. cerebellum V; L.VI, L. cerebellum VI; R.Crus I, R. cerebellum crus I; R.DAC, R. dorsal anterior cingulate; R.frPole, R. frontal pole; R.ICC, R. intracalcarine cortex; R.IFG, R. inferior frontal gyrus, precentral gyrus; R.MFG, R. middle frontal gyrus; R.pCun, R. precuneus; R.STG, R. superior temporal gyrus; R.Thal, R. thalamus. Network-based statistic (NBS) analysis identified eight networks using a t-threshold $=6$ and $P$-value $<0.01$. See Table 2 for list of connections and $t$-statistics.

from $12.6 \pm 2.3$ to $7.0 \pm 2.7$ (5.6 \pm 3.3 (44.2\%) improvement; $t=11.0$, $P<0.001)$. Pre-to-post-waitlist there was little change in YBOCS (pre-waitlist: $25.6 \pm 4.9$, post-waitlist $24.7 \pm 5.4 ; 0.90 \pm 3.1$ (3.5\%) improvement: $t=1.35, P=0.19)$. Patients on average had large improvements in OCD symptoms with CBT, but not waitlist, and compulsions improved more than obsessions (Supplementary Table 2).

Pre-to-post-CBT connectivity changes

Longitudinally, there were extensive, high-magnitude network connectivity increases within the OCD sample, in a largely anterior-posterior pattern. Eight networks exhibited stronger connectivity pre-to-post-CBT (Figure 2, Table 2). As predicted, this enhanced connectivity involved networks with nodes both within classical CSTC circuits (caudate and putamen, dorsal ACC, thalamus), and outside these circuits (including inferior frontal gyrus, middle frontal gyrus, precentral gyrus, insula, posterior cingulate, occipital cortex (intracalcarine cortex, lingual gyrus, lateral occipital cortex) and cerebellum (crus I, V, VI)). Notably, the strongest increases involved cerebellum $\mathrm{V}$ connectivity with middle frontal gyrus, cerebellum Crus I with frontal pole, cerebellum VI to caudate, and cerebellum Crus I to putamen. All eight NBS significant networks involved increases in connectivity 


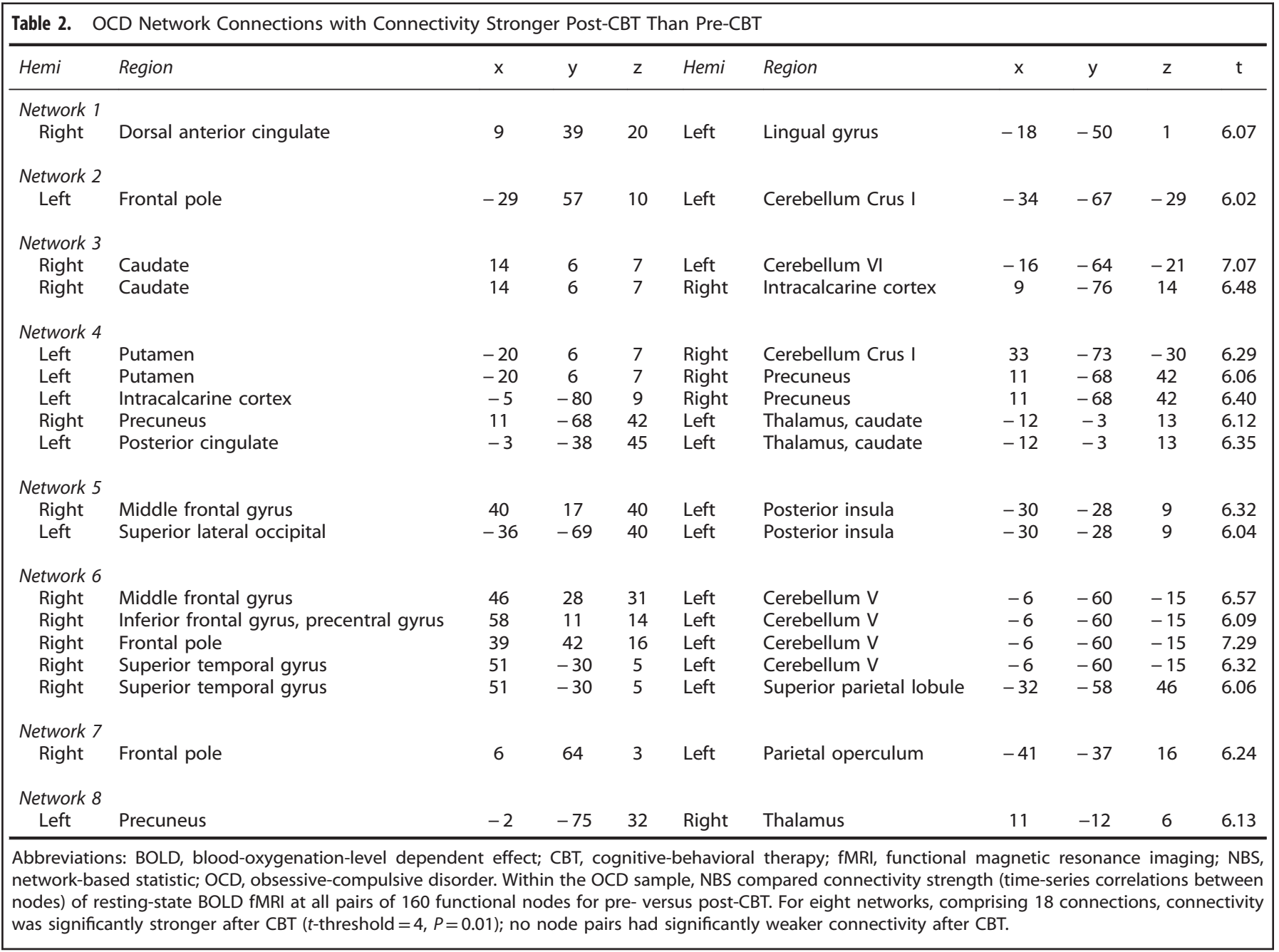

that crossed networks for the previously defined Dosenbach node set: cingulo-opercular, fronto-parietal, default mode, sensorimotor, occipital and cerebellum (Supplementary Figure 4). ${ }^{41}$ No networks exhibited decreased connectivity pre-to-post-CBT.

Pre-to-post-waitlist and pre-to-post-4 week healthy control connectivity changes

There were no significant pre-to-post-waitlist differences in connectivity, indicating no significant effects in OCD participants of being enrolled for 4 weeks with minimal therapist contact. Nor were there any significant differences in connectivity following the passage of 4 weeks of time in healthy controls (Supplementary Figure 5).

Changes in connectivity associated with changes in OCD symptoms

There were no significant associations between changes in YBOCS total scores and changes in connection strength in the eight networks that significantly changed pre-to-post-CBT. Motivated by the increases observed in cerebellar connectivity with caudate/ putamen and frontal pole, we explored post hoc whether these connectivity changes reflected improved control over motor behaviors and mental compulsions, given the strong behavioral emphasis of the ERP treatment. We therefore examined correlations between connectivity changes and YBOCS subscores indexing resistance to compulsions (Item \#9) and control over compulsions
(Item \#10). Increased connectivity between right frontal pole and left parietal operculum was significantly associated with increased efforts to resist compulsions $(P<0.001$ Bonferroni-corrected, Figure 3a). R and p-values for the correlations for Item \#9 and Item \#10 can be found in Supplementary Table 4. Exploratory analyses tested associations between HAMA and MADRS and increases in connectivity; neither survived correction for multiple comparisons.

OCD vs control connectivity pre- and post-CBT

Pre-CBT there were no significant differences in connectivity between OCD and controls, apart from a trend for stronger connectivity in controls between left intraparietal lobule and left precentral gyrus (Supplementary Figure 6). Post-CBT, there were 4 networks with stronger connectivity in OCD than controls (Supplementary Figure 7, Supplementary Table 3, Supplementary Video 2). These included connections between left cerebellum (V) and right and left precentral and postcentral gyri; right cerebellum (VI, Vermis $\mathrm{VI}$ ) with left angular gyrus; and right intracalcarine cortex with right and left caudate and paracingulate.

Pre-CBT connectivity associations with OCD symptoms

Pre-treatment, the network consisting of the connection between left and right anterior insula was significantly associated with Total YBOCS score $(r=-0.35, P=0.02$, Figure 3b). See Supplementary Figure 8 for pre-CBT strength association with HAMA improvement. 

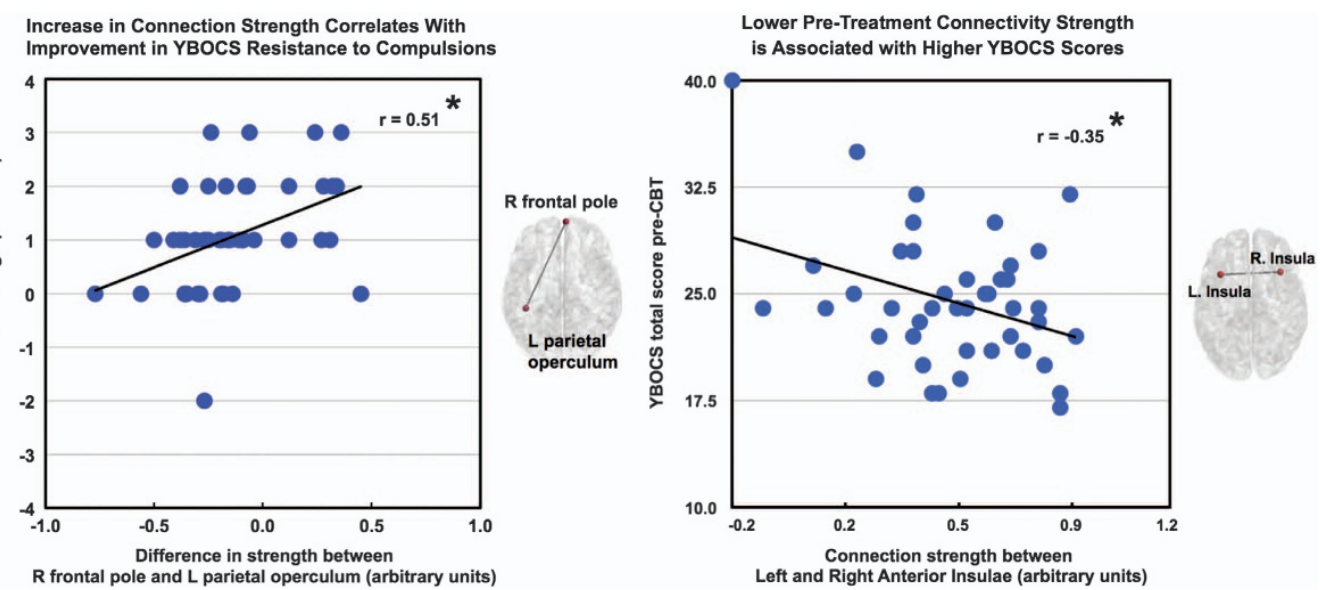

Figure 3. Associations between functional connectivity and obsessive compulsive disorder (OCD) symptom severity. (a) Correlation across OCD participants (blue circles) between pre-to-post-CBT change in OCD symptom severity (YBOCS Item 9 sub-score, resistance to compulsions) and pre-to-post-CBT change in functional connectivity between right frontal pole and left parietal operculum (inset right; 10$\mathrm{mm}$ nodes centered at MNI coordinates $(6,64,3)$ and $(-41,-37,16) ; r=0.51, P<0.001)$. Increased connectivity between this node pair may reflect improved resistance to OCD compulsions induced by CBT. (b) Pre-CBT there was a significant negative correlation between OCD symptom severity (YBOCS scores) and connection strength between left and right insula (inset right). (Nodes centered at (38, 21, -1$)$ and ( -36 , $18,2) ; r=-0.35, P=0.02$ ). Lower pretreatment connectivity between this node pair is associated with worse symptoms. CBT, cognitivebehavioral therapy.

\section{CSTC nodal connectivity results}

For testing of connectivity hypotheses for OCD vs HC between ipsilateral caudate nuclei and OFC, we did not find significant differences pretreatment $(P=0.20)$ or post-treatment $(P=0.55)$. However, in OCD there was a trend for decreased connectivity between left caudate to left orbital frontal cortex from pre- to postCBT $(P=0.052)$. Results of exploratory connectivity analysis for all other node pairs can be found in the Supplementary Materials.

\section{DISCUSSION}

We examined the effects of CBT for OCD on brain functional connectivity for, we believe, the first time using NBS, which allows whole-brain evaluation of network connectivity. Our analysis revealed several novel findings. CBT resulted in increased restingstate functional connectivity in multiple networks within and outside classic CSTC circuits, all of which crossed previously defined, canonical functional connectivity networks. ${ }^{41}$ The strongest increases involved cerebellar connections to striatum and prefrontal cortices. In addition, increased functional connectivity between right frontal pole-left parietal operculum correlated positively with increased ability to resist compulsions. These findings imply that CBT for OCD encompasses neurophysiologic changes in both classical CSTC and non-CSTC pathways. This may signify enhancement of cross-network communication, perhaps involving compensatory mechanisms, beyond simple reversal of pretreatment abnormalities.

The first major finding was that, as predicted, functional connectivity increased in multiple networks from pre- to postCBT in OCD. There were no significant changes in connectivity preto-post-waitlist, or in healthy controls before and after 4 weeks, implying that the observed changes were CBT-related rather than due to nonspecific effects of the passage of time, minimal contact with a therapist, or being scanned twice. Thus, CBT may induce not only global, ${ }^{21}$ but also regional brain network changes in OCD. A prior study ${ }^{20}$ similarly found both global and regional functional connectivity effects of SRI-treatment for OCD. As these effects overlapped only partially with present findings (in occipital cortex, insula, posterior cingulate, superior parietal lobule, middle frontal gyrus, precentral gyrus, but not in cerebellum, caudate, putamen, thalamus, precuneus or ACC), CBT and SRIs may support recovery from OCD along partly distinct pathways.

The strongest increases in connectivity involved connections between cerebellum and striatum and cerebellum and prefrontal cortex. Multiple previous studies in OCD have found abnormal activity or connectivity involving the cerebellum. One fMRI study found that activity during a Stroop task increased in left cerebellar declive, right cerebellar tonsil, and right precuneus with $\mathrm{CBT} \cdot{ }^{42} \mathrm{~A}$ recent study in OCD found abnormal functional connectivity involving cerebellar regions and striatum (caudate, putamen), pallidum, and thalamus; ${ }^{43}$ these increased cerebellar-striatal connections might be a signature of an underlying compensatory process, which, in the context of the current study (although we did not explore modular connectivity), might be enhanced with CBT. Several other cross-sectional studies have found abnormal resting-state functional connectivity in the cerebellum. One found stronger connectivity compared with controls in posterior cerebellum VI, vermis VIIla, IX, X and Crus I. ${ }^{26}$ Similarly, other studies found greater global brain connectivity in OCD than controls in cerebellum (left ${ }^{10}$ and greater connectivity between lateral and inferior cerebellar regions. ${ }^{14}$ In contrast, one study found below-normal functional connectivity in cerebellum. ${ }^{27}$ Another found reduced cerebellar response during fear extinction recall in OCD; in addition, cerebellum and insula activity significantly predicted OCD symptom severity. ${ }^{44}$ Finally, one investigation found elevated regional homogeneity (ReHo) in cerebellum. ${ }^{45}$ In summary, mounting evidence points to cerebellar activity and connectivity playing a role in OCD pathophysiology and/or treatment.

In our study, several connectivity changes involving cerebellum resemble specific effects of CBT seen in other disorders. A study of $\mathrm{CBT}$ for ADHD observed increased functional connectivity with the posterior cerebellum, ${ }^{46}$ implicated in cognitive and emotional tasks $^{47}$ and found increases in connectivity with middle frontal gyrus and superior parietal cortex, similar to the present investigation. A study of CBT for chronic pain found increased local BOLD fluctuations ${ }^{48,49}$ in posterior cingulate and anterior cerebellum (IV, V). ${ }^{50} \mathrm{~A}$ study of CBT for schizophrenia associated stronger dorsolateral-to-cerebellar connectivity with greater clinical improvement. ${ }^{51}$ This suggests some common effects of CBT across different applications. 
Post-CBT increases in cerebellar connectivity to striatum and prefrontal cortex in OCD could represent improved control over motor behaviors and over affect and cognition, respectively. In the current study, cerebellar connectivity increases involved both anterior (V) 'sensorimotor' and posterior (Crus I, VI) 'cognitive and emotional' lobes. ${ }^{47}$ The cerebellum has a role in motor functions, from movement coordination to response-inhibition, ${ }^{47,52}$ and is instrumental in acquisition of goal-directed behavior and optimization of motor responses. ${ }^{53,54}$ In addition, the dysmetria of thought theory ${ }^{55}$ posits cerebellar modulatory involvement in cognition and emotion. Moreover, CBT-relevant effects may be mediated by the vermis through formation of new unconditioned-conditioned associations in fear memory to enable appropriate responses to new stimuli and situations. ${ }^{56}$ Although much remains to be elucidated, current views on functional anatomy in conjunction with previous OCD neuroimaging are consistent with the cerebellum having a key role in CBT-response.

Several other sites exhibited CBT-associated increases in functional connectivity in our study. Among these, two CSTCregions-dorsal ACC and thalamus-were implicated in our earlier PET work on intensive CBT for OCD. ${ }^{57}$ As hypothesized, we found results in classical (caudate, putamen, dorsal ACC and thalamus) and non-classical (inferior frontal gyrus, middle frontal gyrus, precentral gyrus, insula, posterior cingulate cortex and occipital cortex) OCD sites. Some connectivity increases involved the frontal pole, a region where we previously observed abnormal pretreatment functional connectivity in pediatric $O C D^{58}$ and where others $^{59}$ found associations between functional connectivity and compulsive symptoms in adult OCD.

A second (exploratory) finding was that connectivity in one network correlated with increased resistance against compulsions. This suggests enhanced resistance as a prime mechanism of CBT. The type of CBT administered in our trial $\left(E R P^{39}\right)$ is indeed predicated on the patient's ability to resist compulsions. After short-term ERP (conducted here) improvements in compulsions typically exceed improvements in obsessions, ${ }^{60}$ as observed in the present study (44.2\% vs $33.4 \%)$. Increased connectivity at frontal and parietal sites in this study may be associated with improved resistance to compulsions. Improvements in obsessions, which usually catch up longer term, ${ }^{61}$ in contrast, may correspond with normalization of hyperactive CSTC circuits seen in other studies.

A third finding was that, post-CBT, functional connectivity between several sites was higher in OCD than in the control group. There were no significant connectivity differences between OCD and healthy controls pretreatment (differences in one connection between left inferior parietal lobule and left precentral gyrus were evident only for lenient statistical thresholding). The additional connectivity analysis testing CTSC regions-of-interest using nodes derived from a meta-analysis of obsessive compulsive studies, again revealed no significant differences between the OCD and control groups. Thus, two different functional connectivity analyses support the idea that connectivitystrengthening processes may reflect effects of CBT independent of normalization of pathophysiological circuits. Only one connection (right caudate-right intracalcarine cortex) in a network that increased pre-to-post-CBT overlapped with a connection that was significantly greater in OCD than controls post-CBT, although several of the same nodes were involved in both pre-vs-post and OCD-vs-control findings, including ACC, occipital cortex, caudate, thalamus, parietal operculum and precentral gyrus. Additional regions (superior parietal lobule, angular gyrus, postcentral gyrus and supplementary motor area) involved in the latter, supports CBT-effects being neuroanatomically more widespread than previously imagined.

Pre-treatment, OCD symptom severity was associated with connectivity between right and left insula. Similarly, two other studies found strong associations between OCD symptoms and insula. One rsfMRI study found a strong correlation between right insula intrinsic neural activity (amplitude of low-frequency fluctuations-ALFF) and YBOCS. ${ }^{62}$ As mentioned, another study found that insula activity strongly predicted OCD symptom severity. ${ }^{44}$ In the current study, the absence of strong connectivity differences between OCD and controls at baseline is somewhat surprising. An important distinction from other studies is that we used NBS to probe network connectivity across the whole-brain; NBS detects aberrations or changes in networks (clusters or sets of interconnected nodes) ${ }^{17}$ rather than connectivity to/from single nodes or voxels as in previous cross-sectional OCD connectivity studies. ${ }^{10,26,63}$

Further, as OCD is heterogeneous in symptom domains, severity, comorbidities, and neurocognitive dysfunction, ${ }^{64}$ pretreatment variation across patients in individual symptoms (and compensatory 'backup' processes) and individual connectivity biomarkers may obscure group-mean connectivity differences. If, however, intensive CBT more consistently engages one specific set of symptoms across patients (for example, resisting compulsive behaviors) over common network mechanisms, that could lead to appreciable group-wise pre-to-post-CBT increases in connectivity, as well as post-CBT differences in connectivity between OCD and controls, as observed in this study.

A limitation of this study is that the functional node set, chosen for comparison with other investigations, ${ }^{15,41,58,65}$ did not include the amygdala, which has a possible role in aberrant OCD connectivity $^{66}$ and CBT response prediction. ${ }^{67}$ While we included both medicated and unmedicated OCD participants, medicated patients were on stable doses and we used medication status as a covariate for comparisons with controls. Finally, the sample size was larger for the OCD than for the control group; thus power was lower for between-group than for pre-post-CBT comparisons.

In conclusion, CBT increased functional connectivity in multiple networks within and outside CSTC circuits in OCD. Increased cerebellar to striatal and prefrontal connectivity may reflect CBT strengthening the ability to resist compulsions and the acquisition of new non-compulsive goal-directed behaviors and thought patterns. Mechanisms of CBT, at least immediately following intensive treatment, may involve cross-network strengthening of compensatory processes rather than normalizing of pathophysiological circuits. These findings have implications for identifying potential targets for enhancing treatment efficacy (via behavioral, pharmacological and/or brain stimulation treatments) and for monitoring clinical progress in individual patients.

\section{CONFLICT OF INTEREST}

The authors declare no conflict of interest.

\section{ACKNOWLEDGMENTS}

This work was supported by the National Institute of Mental Health, R01MH085900 to JON and JDF. Internal Review Board (IRB) or Animal Use and Care Committee (AUCC) Approval: Approval date and IRB number: IRB \#11-001327, approved 21 June 2011. Trial Registration: ClinicalTrials.gov Identifier: NCT01368510.

\section{AUTHOR CONTRIBUTIONS}

TDM and JDF had full access to all the data in the study and take responsibility for the integrity of the data and the accuracy of the data analysis. From the Department of Psychiatry and Biobehavioral Sciences at UCLA. Study concept and design: TDM, JON, JDF. Acquisition, analysis, or interpretation of data: all authors. Drafting of the manuscript: all authors. Statistical analysis: TDM, JDF. Obtained funding: JON, JDF. Administrative, technical and assessment support: all authors. 


\section{REFERENCES}

1 American Psychiatric Association. Diagnostic and Statistical Manual of Mental Disorders: DSM-5. 5th edn, American Psychiatric Association: Washington, DC, USA, 2013.

2 Markarian Y, Larson MJ, Aldea MA, Baldwin SA, Good D, Berkeljon A et al. Multiple pathways to functional impairment in obsessive-compulsive disorder. Clin Psychol Rev. 2010; 30: 78-88.

3 Eddy KT, Dutra L, Bradley R, Westen D. A multidimensional meta-analysis of psychotherapy and pharmacotherapy for obsessive-compulsive disorder. Clin Psychol Rev. 2004; 24: 1011-1030.

4 Rosa-Alcazar Al, Sanchez-Meca J, Gomez-Conesa A, Marin-Martinez F. Psychological treatment of obsessive-compulsive disorder: a meta-analysis. Clin Psychol Rev. 2008; 28: 1310-1325.

5 Rosa-Alcazar Al, Sanchez-Meca J, Rosa-Alcazar A, Iniesta-Sepulveda M, OlivaresRodriguez J, Parada-Navas JL. Psychological treatment of obsessive-compulsive disorder in children and adolescents: a meta-analysis. Span J Psychol. 2015; 18: E20.

6 Bystritsky A, Munford PR, Rosen RM, Martin KM, Vapnik T, Gorbis EE et al. A preliminary study of partial hospital management of severe obsessive-compulsive disorder. Psychiatr Serv. 1996; 47: 170-174.

7 Calvocoressi L, McDougle Cl, Wasylink S, Goodman WK, Trufan SJ, Price LH. Inpatient treatment of patients with severe obsessive-compulsive disorder. Hosp Community Psychiatry. 1993; 44: 1150-1154.

8 Maia TV, Cooney RE, Peterson BS. The neural bases of obsessive-compulsive disorder in children and adults. Dev Psychopathol. 2008; 20: 1251-1283.

9 Menzies L, Chamberlain SR, Laird AR, Thelen SM, Sahakian BJ, Bullmore ET. Integrating evidence from neuroimaging and neuropsychological studies of obsessive-compulsive disorder: the orbitofronto-striatal model revisited. Neurosci Biobehav Rev. 2008; 32: 525-549.

10 Anticevic A, Hu S, Zhang S, Savic A, Billingslea E, Wasylink S et al. Global restingstate functional magnetic resonance imaging analysis identifies frontal cortex, striatal, and cerebellar dysconnectivity in obsessive-compulsive disorder. Biol Psychiatry. 2014; 75: 595-605.

11 Fitzgerald KD, Stern ER, Angstadt M, Nicholson-Muth KC, Maynor MR, Welsh RC et al. Altered function and connectivity of the medial frontal cortex in pediatric obsessive-compulsive disorder. Biol Psychiatry. 2010; 68: 1039-1047.

12 Milad MR, Rauch SL. Obsessive-compulsive disorder: beyond segregated corticostriatal pathways. Trends Cogn Sci. 2012; 16: 43-51.

13 Stern ER, Fitzgerald KD, Welsh RC, Abelson JL, Taylor SF. Resting-State Functional Connectivity between Fronto-Parietal and Default Mode Networks in ObsessiveCompulsive Disorder. Plos One 2012; 7: e36356.

14 Zhang T, Wang J, Yang Y, Wu Q, Li B, Chen L et al. Abnormal small-world architecture of top-down control networks in obsessive-compulsive disorder. J Psychiatry Neurosci. 2011; 36: 23-31.

15 Shin DJ, Jung WH, He Y, Wang J, Shim G, Byun MS et al. The effects of pharmacological treatment on functional brain connectome in obsessive-compulsive disorder. Biological Psychiatry. 2014; 75: 606-614.

16 Feusner JD, Moody T, Lai TM, Sheen C, Khalsa S, Brown J et al. Brain connectivity and prediction of relapse after cognitive-behavioral therapy in obsessivecompulsive disorder. Front Psychiatry 2015; 6: 74.

17 Zalesky A, Fornito A, Bullmore ET. Network-based statistic: identifying differences in brain networks. Neuroimage 2010; 53: 1197-1207.

18 Goldin PR, Ziv M, Jazaieri H, Hahn K, Heimberg R, Gross JJ. Impact of cognitive behavioral therapy for social anxiety disorder on the neural dynamics of cognitive reappraisal of negative self-beliefs: randomized clinical trial. JAMA Psychiatry 2013; 70: 1048-1056.

19 Mansson KN, Carlbring P, Frick A, Engman J, Olsson CJ, Bodlund O et al. Altered neural correlates of affective processing after internet-delivered cognitive behavior therapy for social anxiety disorder. Psychiatry Res. 2013; 214: 229-237.

20 Månsson KNT, Frick A, Boraxbekk CJ, Marquand AF, Williams SCR, Carlbring P et al. Predicting long-term outcome of Internet-delivered cognitive behavior therapy for social anxiety disorder using fMRI and support vector machine learning. Translational Psychiatry 2015; 5: e530.

21 Mason L, Peters ER, Dima D, Williams SC, Kumari V. Cognitive Behavioral Therapy Normalizes Functional Connectivity for Social Threat in Psychosis. Schizophr Bull. 2016; 42: 684-692.

22 Simmons AN, Norman SB, Spadoni AD, Strigo IA. Neurosubstrates of remission following prolonged exposure therapy in veterans with posttraumatic stress disorder. Psychother Psychosom. 2013; 82: 382-389.

23 Straube B, Lueken U, Jansen A, Konrad C, Gloster AT, Gerlach AL et al. Neural correlates of procedural variants in cognitive-behavioral therapy: a randomized, controlled multicenter FMRI study. Psychother Psychosom. 2014; 83: 222-233.

24 Lazaro L, Calvo A, Ortiz AG, Ortiz AE, Morer A, Moreno E et al. Microstructural brain abnormalities and symptom dimensions in child and adolescent patients with obsessive-compulsive disorder: a diffusion tensor imaging study. Depression and anxiety 2014; 31: 1007-1017.
25 Wen SL, Cheng MF, Cheng MH, Yue JH, Li JF, Xie LJ. Neurocognitive dysfunction and regional cerebral blood flow in medically naive patients with obsessivecompulsive disorder. Developmental neuropsychology 2014; 39: 37-50.

26 Tian L, Meng C, Jiang Y, Tang Q, Wang S, Xie X et al. Abnormal functional connectivity of brain network hubs associated with symptom severity in treatmentnaive patients with obsessive-compulsive disorder: A resting-state functional MRI study. Progress in Neuro-Psychopharmacology and Biological Psychiatry 2016; 66: 104-111.

27 Hou J-M, Zhao M, Zhang W, Song L-H, Wu W-J, Wang J et al. Resting-state functional connectivity abnormalities in patients with obsessive-compulsive disorder and their healthy first-degree relatives. Journal of Psychiatry \& Neuroscience : JPN 2014; 39: 304-311.

28 Harrison BJ, Soriano-Mas C, Pujol J, Ortiz H, Lopez-Sola M, Hernandez-Ribas R et al. Altered corticostriatal functional connectivity in obsessive-compulsive disorder. Arch Gen Psychiatry. 2009; 66: 1189-1200.

29 Sakai Y, Narumoto J, Nishida S, Nakamae T, Yamada K, Nishimura T et al. Corticostriatal functional connectivity in non-medicated patients with obsessivecompulsive disorder. Eur Psychiatry. 2011; 26: 463-469.

30 DiNardo PA, Brown TA, Barlow DH. Anxiety Disorders Interview Schedule for DSM-IV: Lifetime version. Graywind: Albany, 1994.

31 Goodman WK, Price LH, Rasmussen SA, Mazure C, Fleischmann RL, Hill CL et al. The Yale-Brown Obsessive Compulsive Scale .1. Development, Use, and Reliability. Arch Gen Psychiatry. 1989; 46: 1006-1011.

32 Wechsler D. Wechsler Abbreviated Scale of Intelligence (WASI). Psychological Corporation: San Antonio, 1999.

33 Pocock SJ. Interim analyses for randomized clinical trials: the group sequential approach. Biometrics 1982; 38: 153-162.

34 Smoak C, Lin J-SA SAS program to perform adaptive randomizationStatistics, Data Analysis, and Data Mining. Chiron Corporation: Emeryville, CA, 2006.

35 Foa EB, Huppert JD, Leiberg S, Langner R, Kichic R, Hajcak G et al. The ObsessiveCompulsive Inventory: development and validation of a short version. Psychol Assess 2002; 14: 485-496.

36 Hamilton M. The assessment of anxiety states by rating. The British journal of medical psychology 1959; 32: 50-55.

37 Montgomery SA, Asberg M. A new depression scale designed to be sensitive to change. Br J Psychiatry. 1979; 134: 382-389.

38 Endicott J, Spitzer RL, Fleiss JL, Cohen J. The global assessment scale. A procedure for measuring overall severity of psychiatric disturbance. Arch Gen Psychiatry. 1976; 33: 766-771.

39 Kozak MJ, Foa EB. Mastery of obsessive-compulsive disorder: a cognitive-behavioral approach client workbook. Oxford University Press: New York, 1997.

40 Power JD, Barnes KA, Snyder AZ, Schlaggar BL, Petersen SE. Spurious but systematic correlations in functional connectivity MRI networks arise from subject motion. Neuroimage 2012; 59: 2142-2154.

41 Dosenbach NU, Nardos B, Cohen AL, Fair DA, Power JD, Church JA et al. Prediction of individual brain maturity using fMRI. Science 2010; 329: 1358-1361.

42 Nabeyama M, Nakagawa A, Yoshiura T, Nakao T, Nakatani E, Togao $O$ et al. Functional MRI study of brain activation alterations in patients with obsessivecompulsive disorder after symptom improvement. Psychiatry Res. 2008; 163: 236-247.

43 Vaghi MM, Vértes PE, Kitzbichler MG, Apergis-Schoute AM, van der Flier FE, Fineberg NA et al. Specific Frontostriatal Circuits for Impaired Cognitive Flexibility and Goal-Directed Planning in Obsessive-Compulsive Disorder: Evidence From Resting-State Functional Connectivity. Biological Psychiatry 2017; 81: 708-717.

44 Milad MR, Furtak SC, Greenberg JL, Keshaviah A, Im JJ, Falkenstein MJ et al. Deficits in conditioned fear extinction in obsessive-compulsive disorder and neurobiological changes in the fear circuit. JAMA Psychiatry 2013; 70: 608-618, quiz 554.

45 Ping L, Su-Fang L, Hai-Ying H, Zhang-Ye D, Jia L, Zhi-Hua G et al. Abnormal Spontaneous Neural Activity in Obsessive-Compulsive Disorder: A Resting-State Functional Magnetic Resonance Imaging Study. PLOS ONE 2013; 8: e67262.

46 Wang X, Cao Q, Wang J, Wu Z, Wang P, Sun L et al. The effects of cognitivebehavioral therapy on intrinsic functional brain networks in adults with attentiondeficit/hyperactivity disorder. Behaviour Research and Therapy. 2016; 76: 32-39.

47 Stoodley CJ, Schmahmann JD. Functional topography in the human cerebellum: a meta-analysis of neuroimaging studies. Neuroimage 2009; 44: 489-501.

48 Zou QH, Zhu CZ, Yang Y, Zuo XN, Long XY, Cao QJ et al. An improved approach to detection of amplitude of low-frequency fluctuation (ALFF) for resting-state fMRI: fractional ALFF. J Neurosci Methods. 2008; 172: 137-141.

49 Zuo XN, Di Martino A, Kelly C, Shehzad ZE, Gee DG, Klein DF et al. The oscillating brain: complex and reliable. Neuroimage 2010; 49: 1432-1445.

50 Shpaner M, Kelly C, Lieberman G, Perelman H, Davis M, Keefe FJ et al. Unlearning chronic pain: A randomized controlled trial to investigate changes in intrinsic brain connectivity following Cognitive Behavioral Therapy. Neurolmage: Clinical 2014; 5: 365-376. 
51 Kumari V, Peters ER, Fannon D, Antonova E, Premkumar P, Anilkumar AP et al. Dorsolateral prefrontal cortex activity predicts responsiveness to cognitivebehavioral therapy in schizophrenia. Biol Psychiatry. 2009; 66: 594-602.

52 Manni E, Petrosini L. A century of cerebellar somatotopy: a debated representation. Nat Rev Neurosci. 2004; 5: 241-249.

53 Burguiere E, Arabo A, Jarlier F, De Zeeuw Cl, Rondi-Reig L. Role of the cerebellar cortex in conditioned goal-directed behavior. J Neurosci. 2010; 30: 13265-13271.

54 Burguiere E, Arleo A, Hojjati M, Elgersma Y, De Zeeuw Cl, Berthoz A et al. Spatial navigation impairment in mice lacking cerebellar LTD: a motor adaptation deficit? Nat Neurosci. 2005; 8: 1292-1294.

55 Schmahmann JD. From movement to thought: anatomic substrates of the cerebellar contribution to cognitive processing. Hum Brain Mapp. 1996; 4: 174-198.

56 Sacchetti B, Scelfo B, Strata P. Cerebellum and emotional behavior. Neuroscience 2009; 162: 756-762.

57 Saxena S, Gorbis E, O'Neill J, Baker SK, Mandelkern MA, Maidment KM et al. Rapid effects of brief intensive cognitive-behavioral therapy on brain glucose metabolism in obsessive-compulsive disorder. Mol Psychiatry. 2008; 14: 197-205.

58 Armstrong CC, Moody TD, Feusner JD, McCracken JT, Chang S, Levitt JG et al. Graph-theoretical analysis of resting-state $\mathrm{fMRI}$ in pediatric obsessive-compulsive disorder. J Affect Disord. 2016; 193: 175-184.

59 Meunier D, Ersche KD, Craig KJ, Fornito A, Merlo-Pich E, Fineberg NA et al. Brain functional connectivity in stimulant drug dependence and obsessive-compulsive disorder. Neuroimage 2012; 59: 1461-1468

60 Lindsay M, Crino R, Andrews G. Controlled trial of exposure and response prevention in obsessive-compulsive disorder. The British Journal of Psychiatry 1997; 171: 135-139.

61 Nakatani E, Nakagawa A, Nakao T, Yoshizato C, Nabeyama M, Kudo A et al. A randomized controlled trial of Japanese patients with obsessive-compulsive disorder--effectiveness of behavior therapy and fluvoxamine. Psychother Psychosom. 2005; 74: 269-276.
62 Zhu Y, Fan Q, Zhang H, Qiu J, Tan L, Xiao Z et al. Altered intrinsic insular activity predicts symptom severity in unmedicated obsessive-compulsive disorder patients: a resting state functional magnetic resonance imaging study. BMC psychiatry 2016; 16: 104.

63 Beucke JC, Sepulcre J, Talukdar T et al. Abnormally high degree connectivity of the orbitofrontal cortex in obsessive-compulsive disorder. JAMA Psychiatry 2013; 70: 619-629.

64 Lochner C, Stein DJ. Heterogeneity of obsessive-compulsive disorder: a literature review. Harvard review of psychiatry 2003; 11: 113-132.

65 Dosenbach NU, Fair DA, Miezin FM, Cohen AL, Wenger KK, Dosenbach RA et al. Distinct brain networks for adaptive and stable task control in humans. Proc Nat Acad Sci U S A. 2007; 104: 11073-11078.

66 van Velzen LS, de Wit SJ, Curcic-Blake B, Cath DC, de Vries FE, Veltman DJ et al Altered Inhibition-Related Frontolimbic Connectivity in Obsessive-Compulsive Disorder. Hum Brain Mapp. 2015; 36: 4064-4075.

67 Gottlich M, Kramer UM, Kordon A, Hohagen F, Zurowski B. Resting-state connectivity of the amygdala predicts response to cognitive behavioral therapy in obsessive compulsive disorder. Biol Psychol. 2015; 111: 100-109.

(i) $\$$ This work is licensed under a Creative Commons AttributionNonCommercial-NoDerivs 4.0 International License. The images or other third party material in this article are included in the article's Creative Commons license, unless indicated otherwise in the credit line; if the material is not included under the Creative Commons license, users will need to obtain permission from the license holder to reproduce the material. To view a copy of this license, visit http:// creativecommons.org/licenses/by-nc-nd/4.0/

(c) The Author(s) 2017

Supplementary Information accompanies the paper on the Translational Psychiatry website (http://www.nature.com/tp) 